\title{
High resolution pituitary gland MRI at 7.0 tesla: a clinical evaluation in Cushing's disease
}

\author{
Alexandra A. J. de Rotte ${ }^{1}$ • Amy Groenewegen ${ }^{1}$ • Dik R. Rutgers ${ }^{1}$ • \\ Theo Witkamp ${ }^{1}$ - Pierre M. J. Zelissen ${ }^{2}$ F. J. Anton Meijer ${ }^{3}$. \\ Erik J. van Lindert ${ }^{4} \cdot$ Ad Hermus $^{5}$ - Peter R. Luijten ${ }^{1}$ - Jeroen Hendrikse ${ }^{1}$
}

Received: 22 January 2015 /Revised: 29 March 2015 / Accepted: 16 April 2015 / Published online: 20 May 2015

(C) The Author(s) 2015. This article is published with open access at Springerlink.com

\begin{abstract}
Objective To evaluate the detection of pituitary lesions at $7.0 \mathrm{~T}$ compared to $1.5 \mathrm{~T}$ MRI in 16 patients with clinically and biochemically proven Cushing's disease.

Methods In seven patients, no lesion was detected on the initial 1.5 T MRI, and in nine patients it was uncertain whether there was a lesion. Firstly, two readers assessed both $1.5 \mathrm{~T}$ and 7.0 T MRI examinations unpaired in a random order for the presence of lesions. Consensus reading with a third neuroradiologist was used to define final lesions in all MRIs. Secondly, surgical outcome was evaluated. A comparison was made between the lesions visualized with MRI and the lesions found during surgery in $9 / 16$ patients.

Results The interobserver agreement for lesion detection was good at $1.5 \mathrm{~T}$ MRI $(\kappa=0.69)$ and $7.0 \mathrm{~T}$ MRI $(\kappa=0.62)$. In five patients, both the $1.5 \mathrm{~T}$ and $7.0 \mathrm{~T}$ MRI enabled visualization of
\end{abstract}

Electronic supplementary material The online version of this article (doi:10.1007/s00330-015-3809-x) contains supplementary material, which is available to authorized users.

Alexandra A. J. de Rotte

A.A.J.deRotte@umcutrecht.nl

1 Department of Radiology, University Medical Center Utrecht, Heidelberglaan 100, Postbox 85500, 3508

GA Utrecht, The Netherlands

2 Department of Internal Medicine (Section of Endocrinology), University Medical Center Utrecht, Utrecht, The Netherlands

3 Department of Radiology and Nuclear Medicine, Radboud University Medical Center, Nijmegen, The Netherlands

4 Department of Neurosurgery, Radboud University Medical Center, Nijmegen, The Netherlands

5 Department of Internal Medicine (Section of Endocrinology), Radboud University Medical Center, Nijmegen, The Netherlands a lesion on the correct side of the pituitary gland. In three patients, 7.0 T MRI detected a lesion on the correct side of the pituitary gland, while no lesion was visible at $1.5 \mathrm{~T}$ MRI. Conclusion The interobserver agreement of image assessment for 7.0 T MRI in patients with Cushing's disease was good, and lesions were detected more accurately with $7.0 \mathrm{~T}$ MRI.

Key Points

- Interobserver agreement for lesion detection on 1.5 T MRI was good;

- Interobserver agreement for lesion detection on 7.0 T MRI was good;

- 7.0 Tenabled confirmation of unclear lesions at 1.5 T;

- 7.0 T enabled visualization of lesions not visible at 1.5 T.

Keywords Pituitary gland · Pituitary adenoma $\cdot$ Cushing's disease, pituitary $\cdot$ Pituitary ACTH hypersecretion · Magnetic resonance imaging

\section{Introduction}

Endogenous Cushing's syndrome is a clinical condition in which the adrenal glands secrete excessive amounts of cortisol. In Cushing's disease, which is responsible for $80-85 \%$ of adrenocorticotrophic hormone (ACTH)-dependent Cushing's syndrome, an ACTH producing adenoma located in the pituitary gland stimulates the adrenal glands to secrete the excessive amounts of cortisol [1]. In the majority of cases these adenomas are microadenomas, which are challenging to diagnose [2]. Therefore, final diagnosis is often based on central venous sampling and magnetic resonance imaging (MRI) [3]. In case a pituitary origin of Cushing's syndrome is confirmed, the treatment of choice is surgical removal of the lesion, for which visualization of the lesion is crucial [4-6]. 
Consequently, it is important to visualize the pituitary gland with the highest possible spatial resolution, which enables correct delineation of small anatomical structures and pathological lesions.

In routine clinical practice, MRI is the preferred imaging technique, since it can attain the highest spatial resolution, signal-to-noise ratio (SNR), and contrast-to-noise ratio (CNR) of the pituitary gland [7, 8]. MRI at a field strength of 1.5 Tesla $(\mathrm{T})$ is most commonly used, but pituitary microadenomas remain undetected in 36-63\% of patients at this field strength [9-13]. The higher attainable SNR and spatial resolution at higher field strengths - for instance 3.0 T or 7.0 $\mathrm{T}$ - theoretically has the potential to significantly improve the tumour detection rate $[14,15]$.

Recently, a high resolution 7.0 T pituitary gland MRI protocol with a $0.8 \mathrm{~mm}$ isotropic voxelsize was presented [16]. Feasibility was demonstrated in healthy control subjects and case examples of patients with Cushing's disease were given. The aim of the current preliminary retrospective study is to evaluate the detection of pituitary lesions with high resolution pituitary gland imaging at $7.0 \mathrm{~T}$ compared to $1.5 \mathrm{~T}$ MRI in patients with clinically and biochemically proven Cushing's disease with inconclusive lesions localization at 1.5 T MRI.

\section{Methods}

\section{Subjects}

Between January 2012 and July 2014, 16 patients with clinically and biochemically proven Cushing's disease underwent 7.0 T MRI in the University Medical Center Utrecht. Patients were referred to Utrecht when the standard 1.5 T MRI was inconclusive for the detection of an adenoma. An inconclusive diagnosis was defined as no pathologies visible or as an unclear lesion visible, which both needed further investigation. The institutional review board (IRB) of the University Medical Center Utrecht approved imaging at 7.0 T MRI for clinical purposes in case lower field strength MRI was inconclusive for diagnosis. All patients gave written informed consent. The Radboud University Medical Center referred eight patients to Utrecht and the remaining eight patients were patients from the University Medical Center Utrecht. IRB approval was obtained in both university medical centres to evaluate retrospectively the clinical information and $1.5 \mathrm{~T}$ and 7.0 T MR images of all patients. Data of five patients was previously published as part of clinical case examples of the high resolution pituitary protocol. [16]

\section{Clinical evaluation}

A pituitary origin of ACTH-dependent Cushing's syndrome was established by biochemical tests, including inferior petrosal sinus sampling (IPSS). A detailed description of these tests, used for diagnosis, is given in the Appendix.

\section{Magnetic resonance imaging}

Initial imaging of all patients was performed on $1.5 \mathrm{~T}$ MRI systems, using a local clinical protocol for depicting the pituitary gland. The protocol consisted of a pre- and post-contrast $\mathrm{T}_{1}$-weighted turbo spin echo (TSE) sequence, a dynamic $\mathrm{T}_{1}$ weighted TSE sequence, and a $\mathrm{T}_{2}$-weighted TSE sequence.

Note that five patients were referred from peripheral hospitals. In all patients, the MRI from the peripheral hospital was of good quality and consequently used for the current retrospective analysis. In six patients, the dynamic $T_{1}$-weighted TSE sequence was lacking in the protocol. Also, in six patients, a proton density-weighted sequence and in two patients a diffusion-weighted sequence was added to the protocol.

All 7.0 T MRI examinations were performed on a 7.0 T whole-body MRI system (Philips Healthcare, Cleveland, OH, USA) with a 32-channel receive-coil and a volume transmit/ receive-coil (Nova Medical, Inc., Wilmington, MA, USA). The imaging protocol was similar to the dedicated imaging protocol previously described [16]. Briefly, the protocol consisted of a three-dimensional (3D) $\mathrm{T}_{2}$-weighted TSE sequence and a $3 \mathrm{D} \mathrm{T}_{1}$-weighted magnetization-prepared inversion recovery (MPIR) TSE sequence before and after contrast administration. Scanning parameters are demonstrated in Table 1. A single dose, $0.1 \mathrm{~mL} / \mathrm{kg}$, gadolinium-based contrast agent was used. Post-contrast imaging was started approximately 2 min after contrast injection. A dynamic contrastenhanced sequence was not performed. As described previously, these sequences were used to enable imaging with limited amounts of artefacts [16]. Susceptibility and movement were the most disturbing artefacts due to the anatomical position of the pituitary gland in close proximity to the sphenoid sinus and, respectively, due to the high resolution, in combination with the relatively long duration of the sequences [16].

Table 1 Scanning parameters

\begin{tabular}{lll}
\hline & $\mathrm{T}_{1}$ MPIR TSE & $\mathrm{T}_{2}$ TSE \\
\hline FOV (mm) & $250 \times 250 \times 190$ & $250 \times 250 \times 190$ \\
Acquired resolution $(\mathrm{mm})$ & $0.8 \times 0.8 \times 0.8$ & $0.7 \times 0.7 \times 0.7$ \\
Acquired voxel size $\left(\mathrm{mm}^{3}\right)$ & 0.512 & 0.343 \\
TR/TI (ms) & $3952 / 1375$ & $3200 /-$ \\
TE/equivalent TE (ms) & $37 / 19$ & $300 / 58$ \\
Flip angle (degrees) & 150 & 120 \\
TFE/TSE factor & 158 & 182 \\
NSA & 2 & 2 \\
SENSE factor (AP x RL) & $2 \times 3$ & $2 \times 2.8$ \\
Duration (min:sec) & $10: 40$ & $10: 24$ \\
\hline
\end{tabular}




\section{Image analysis}

Firstly, the images were evaluated by two experienced neuroradiologists (ThW and DR) with 25 and 8 years' experience, respectively. All $1.5 \mathrm{~T}$ and $7.0 \mathrm{~T}$ scans were presented to the observers, unpaired and in a random order. Images were evaluated for the presence and number of lesions in the pituitary gland and the precise location of all identified lesions. Secondly, consensus reading with a third neuroradiologist $(\mathrm{JH})$, with 10 years' experience, was used to evaluate the differences between both first observers and to make a final decision for every MRI. All observers were masked for patient characteristics, clinical and biochemical data, and the initial MRI diagnoses. However, they were not masked for the diagnosis of Cushing's disease.

Interobserver agreement for the presence of lesions was evaluated with Cohen's kappa coefficient. The kappa ranged from 0 (no agreement) to 1 (perfect agreement) and was divided into the following five categories: $<0.20$ (poor), 0.21 to 0.40 (fair), 0.41 to 0.60 (moderate), 0.61 to 0.80 (good), and 0.81 to 1.00 (very good). The statistical analysis was performed in IBM SPSS Statistics version 20 (IBM Corporation, Armonk, NY, USA).

\section{Surgery}

When patients underwent surgery, the macroscopic and microscopic outcome of surgery, symptom status after surgery, and morning serum cortisol levels in the first week after surgery were used to evaluate the clinical outcome of surgery. Cure was defined as a morning serum cortisol of $<0.14 \mu \mathrm{mol} /$ $\mathrm{L}$, based on previous published literature, or as clinical remission [17]. The final results of 1.5 T MRI and 7.0 T MRI were compared with the surgical outcome. Histopathology was not taken into account for the current analysis because the surgical procedure was not standardized, which might result in a poor correlation between pathological outcome and actual remission.

\section{Results}

\section{Subjects}

In total, 16 patients underwent 7.0 T MRI of the pituitary gland. In seven patients, no lesion was detected on the initial 1.5 T MRI, and in nine patients, it was uncertain whether there was a lesion.

\section{Image analysis}

There was absolute agreement between both observers for the results of 1.5 T MRI scans in 11 patients. In five patients, both observers scored the 1.5 T MRI as no pathologies, and in six patients, both observers identified one identical lesion $(n=4)$ or two identical lesions $(n=2)$. In the remaining five patients, one observer identified a lesion, whereas the other observer did not $(n=1)$; both observers identified one identical lesion, and only one observer identified a second lesion $(\mathrm{n}=2)$; or a lesion in the right side of the pituitary gland was identified by one observer, and a lesion in the left side of the pituitary gland was identified by the second observer $(n=2)$.

There was absolute agreement between both observers for the results of 7.0 T MRI scans in 10 patients. In one patient, both observers scored the 7.0 T MRI as no pathologies, and in nine patients, both observers identified one identical lesion $(n=6)$, or two identical lesions $(n=3)$. In the remaining six patients, one observer identified a lesion, whereas the other did not $(n=5)$, or both observers identified one identical lesion, and only one observer identified a second lesion $(\mathrm{n}=1)$. The probability-adjusted interobserver agreement was good for 1.5 T MRI $(\kappa=0.69)$ and for 7.0 T MRI $(\kappa=0.62)$. The results are presented in Table 1, including the initial diagnosis on $1.5 \mathrm{~T}$ MRI.

After consensus reading, 13 lesions, in nine patients, were identified on 1.5 T MRI scans, compared to 17 lesions, in 13 patients, on 7.0 T MRI. In three patients two lesions were visible on both $1.5 \mathrm{~T}$ MRI and 7.0 T MRI. In two patients, two lesions were visible only on $1.5 \mathrm{~T}$ MRI $(\mathrm{n}=1)$ or only on 7.0 T MRI $(\mathrm{n}=1)$. Comparison of the $1.5 \mathrm{~T}$ and $7.0 \mathrm{~T}$ results demonstrated in 10 patients an identical diagnosis on $1.5 \mathrm{~T}$ and 7.0 $\mathrm{T}$. In the remaining six patients, there was discrepancy between $1.5 \mathrm{~T}$ and $7.0 \mathrm{~T}$. The exact results are demonstrated in Table 2.

Examples are given in Figs. 1 and 2. In the patient in Fig. 1, initially no pathologies were identified on $1.5 \mathrm{~T}$ MRI. For the current study, there was discrepancy between both observers for the $1.5 \mathrm{~T}$ MRI, only one observer identified a lesion. On 7.0 T, however, both observers identified this lesion. This case demonstrates that 7.0 T MRI might contribute to a more certain diagnosis.

\section{Surgery}

Transsphenoidal endoscopic microadenomectomy was performed in 13 patients, and one patient underwent transsphenoidal total hypophysectomy. An overview of the outcome of surgery in all patients is demonstrated in Table 1 . In four patients, both sides of the pituitary gland were explored, or the side of exploration was not clearly reported by the neurosurgeon. Since for a correct correlation with MRI, it is crucial to know from which side a potential microadenoma has been excised during surgery, these four patients were not used for further analysis. For the remaining nine patients, the right side of the pituitary gland was explored in seven patients and the left side of the pituitary gland was explored in two 
Table 2 Patient overview with MRI results after consensus

\begin{tabular}{|c|c|c|c|c|c|c|}
\hline \multirow[t]{2}{*}{ Patient } & \multirow{2}{*}{$\begin{array}{l}\text { Initial } \\
\text { diagnosis } \\
1.5 \mathrm{~T}\end{array}$} & \multicolumn{2}{|c|}{ Study analysis } & \multirow[t]{2}{*}{ Surgery } & \multirow{2}{*}{$\begin{array}{l}\text { Morning } \\
\text { serum } \\
\text { cortisol }\end{array}$} & \multirow[t]{2}{*}{ Cure $^{\mathrm{a}}$} \\
\hline & & $1.5 \mathrm{~T}$ & $7.0 \mathrm{~T}$ & & & \\
\hline 1 & $\mathrm{~L}$ & $\mathrm{~N}$ & $\mathrm{~L}$ & Unknown $^{\mathrm{b}}$ & 0.02 & Yes \\
\hline 2 & $\mathrm{~N}$ & $\mathrm{R} / \mathrm{L}$ & $\mathrm{R} / \mathrm{L}$ & $\mathrm{R}$ & 0.05 & Yes \\
\hline 3 & $\mathrm{R}$ & $\mathrm{R}$ & $\mathrm{R}$ & $\mathrm{R}$ & $<0.02$ & Yes \\
\hline 4 & $\mathrm{~L}$ & $\mathrm{~L}$ & $\mathrm{~L}$ & $\mathrm{~L}$ & $<0.02$ & Yes \\
\hline 5 & $\mathrm{R}$ & $\mathrm{N}$ & $\mathrm{N}$ & Both $^{c}$ & 0.2 & Yes \\
\hline 6 & $\mathrm{R}$ & $\mathrm{R}$ & $\mathrm{C}$ & $\mathrm{R}$ & $<0.02$ & Yes \\
\hline 7 & $\mathrm{~N}$ & $\mathrm{~N}$ & $\mathrm{~N}$ & n.a. & n.a. & n.a. \\
\hline 8 & $\mathrm{~N}$ & $\mathrm{~N}$ & $\mathrm{~N}$ & n.a. & n.a. & n.a. \\
\hline 9 & $\mathrm{~L}$ & $\mathrm{~L}$ & $\mathrm{~L}$ & n.a. & n.a. & n.a. \\
\hline 10 & $\mathrm{~N}$ & $\mathrm{~N}$ & $\mathrm{R} / \mathrm{L}$ & $\mathrm{R}$ & $<0.02$ & Yes \\
\hline 11 & $\mathrm{~N}$ & $\mathrm{~N}$ & $\mathrm{R}$ & $\mathrm{R}$ & $<0.02$ & Yes \\
\hline 12 & $\mathrm{~L} / \mathrm{R}$ & $\mathrm{L}$ & $\mathrm{L} / \mathrm{C}$ & Both $^{c}$ & 0.12 & Yes \\
\hline 13 & $\mathrm{~N}$ & $\mathrm{~N}$ & $\mathrm{R}$ & $\mathrm{R}$ & 0.05 & Yes \\
\hline 14 & $\mathrm{~L} / \mathrm{R}$ & $\mathrm{L}$ & $\mathrm{L}$ & Both $^{c}$ & 0.72 & No \\
\hline 15 & $\mathrm{~N}$ & $\mathrm{R} / \mathrm{L}$ & $\mathrm{L}$ & $\mathrm{L}$ & 0.02 & Yes \\
\hline 16 & $\mathrm{R}$ & $\mathrm{R} / \mathrm{C}$ & $\mathrm{R} / \mathrm{C}$ & $\mathrm{R}$ & $<0.02$ & Yes \\
\hline
\end{tabular}

Results are lesions found right $(R)$, left $(L)$, or central $(C)$ in the pituitary gland or no pathologies $(N)$, or a combination of those. $n . a .=$ not applicable, these patients have not had surgery yet; ${ }^{\text {a }}$ Cure is defined as morning serum cortisol of $<0.14 \mu \mathrm{mol} / \mathrm{L}$ or clinical remission; ${ }^{b}$ From these patients, it is unknown from which side of the pituitary gland tissue has been excised; ${ }^{c}$ from these patients, tissue is excised from both sides of the pituitary gland; morning serum cortisol $(\mu \mathrm{mol} / \mathrm{L})$ is determined in the week post-surgery

patients. All nine patients were cured after surgery with a morning serum cortisol level of $<0.14 \mu \mathrm{mol} / \mathrm{L}$ after surgery. Exact values are demonstrated in Table 1. In five patients, both the 1.5 T and 7.0 T MRI enabled visualization of a lesion on the correct side of the pituitary gland. In three patients, $7.0 \mathrm{~T}$
MRI enabled visualization of a lesion on the correct side of the pituitary gland, while no lesion was visible at $1.5 \mathrm{~T}$ MRI. In one patient only, 1.5 T MRI detected the lesion on the correct side of the pituitary gland. In this patient, a different lesion was visualized at 7.0 T MRI.

\section{Discussion}

The current preliminary study demonstrated that the interobserver agreement for the detection of microadenomas in patients with Cushing's disease is good, both at $1.5 \mathrm{~T}$ MRI and 7.0 T MRI. Additionally, in five patients, 7.0 T enabled confirmation of an unclear lesion at $1.5 \mathrm{~T}$, and in three patients, 7.0 T enabled visualization of a lesion not visible at $1.5 \mathrm{~T}$.

Previous literature has demonstrated that with high field strength 3.0 T MRI, an increased detection rate of pituitary lesions is achieved compared to standard 1.0 and 1.5 T MRI $[15,18,19]$. This is important as there is evidence that a precise localization is associated with a more favourable outcome of surgery $[5,20]$. However, other studies could not demonstrate that the cure rate is higher in patients in whom the lesion is actually visualized before surgery $[4,18,21]$. Nevertheless, it is obvious that in patients with no visible lesion on MRI, a more extensive surgical procedure is needed [4].

A high spatial resolution 7.0 T MRI protocol has demonstrated a more detailed visualization of pituitary gland anatomy and promising results for detecting pathology [16]. Furthermore, the current study has demonstrated good interobserver agreement for both 1.5 T MRI and 7.0 T MRI, apart from the limited experience with 7.0 T MRI of both observers. Although the initial diagnosis on $1.5 \mathrm{~T}$ in all patients was inconclusive in the current study, there was absolute
Fig. 1 The $1.5 \mathrm{~T}$ and $7.0 \mathrm{~T}$ MR images of a 56-year-old woman with Cushing's disease. The lesion marked in the $1.5 \mathrm{~T}$ images (white arrowhead) was identified by only one of the two observers. Note that this lesion was only visible on one slice. After consensus with a third observer, the final decision was "no lesions". The lesion marked in the $7.0 \mathrm{~T}$ images (white arrow) was identified by both observers

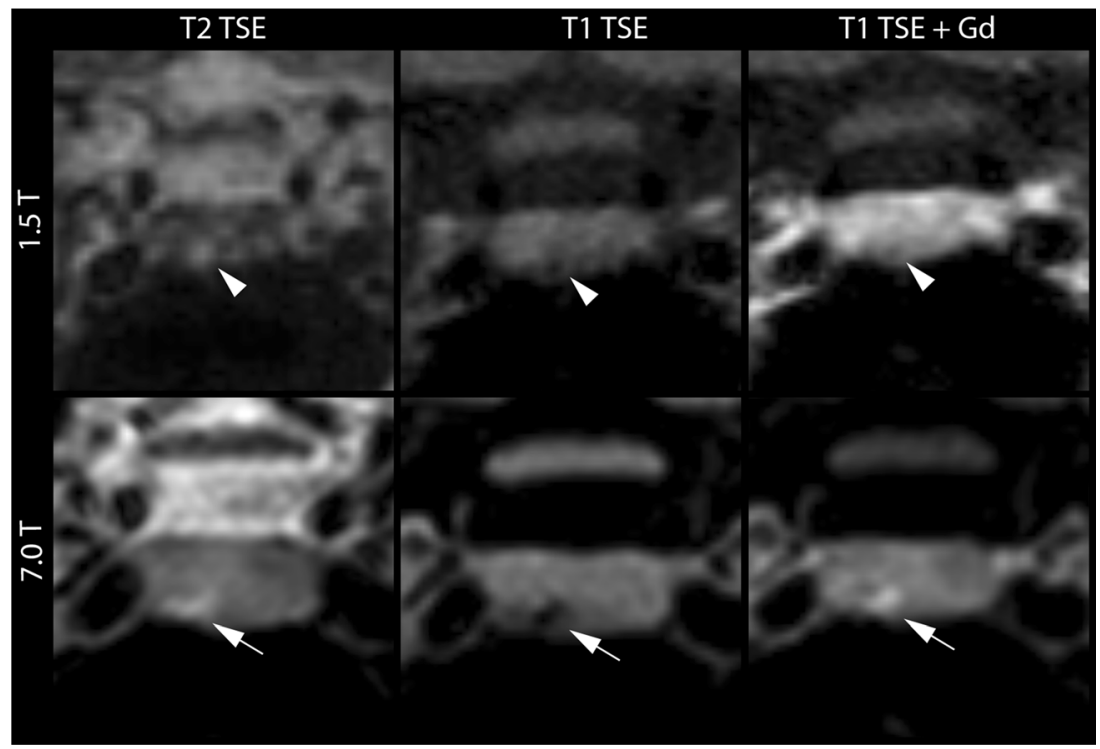


Fig. 2 The transversal 7.0 Tesla $\mathrm{T}_{1}$ MPIR TSE images before and after contrast enhancement of a 42-year-old male diagnosed with Cushing's disease. On the $1.5 \mathrm{~T}$ images, not demonstrated, there was no lesion detected. On 7.0 T MRI there was a lesion detected (arrowhead) in the left side of the pituitary gland. During surgery, an ACTH producing microadenoma was removed from the left side of the pituitary gland

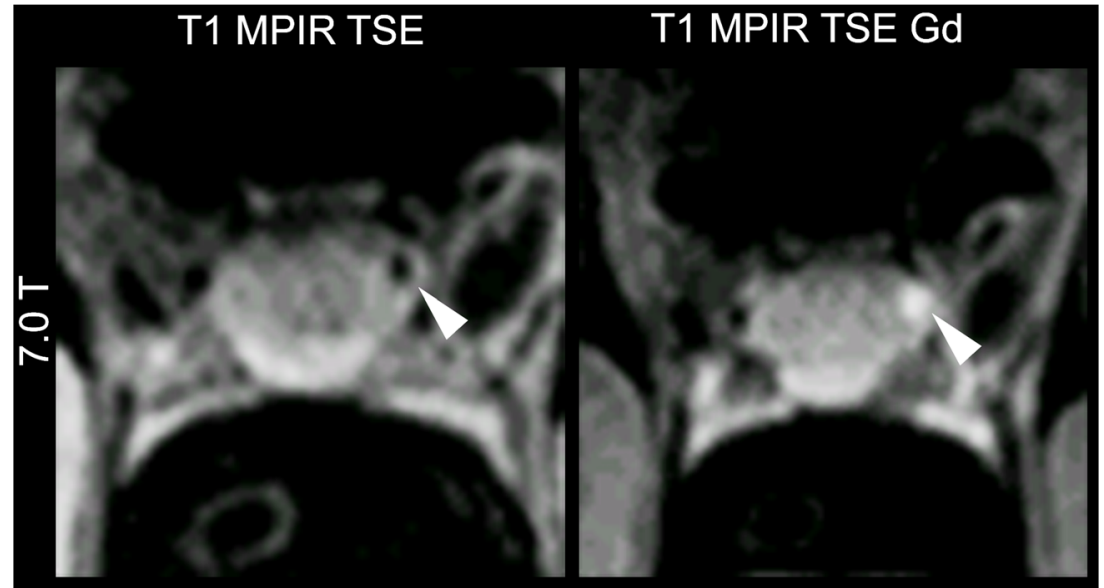

agreement for the diagnosis on those images in 11 patients. This high rate of agreement suggests that consensus reading in clinical practice might also confirm inconclusive diagnoses. However, the high spatial resolution of 7.0 T MRI enables visualization more detail than standard 1.5 T MRI, which might result in more visible lesions.

The current study has demonstrated that high resolution 7.0 T MRI allows visualization of more pituitary lesions compared to $1.5 \mathrm{~T}$ MRI. In the small series of patients presented, the number of lesions visualized with 7.0 T MRI is higher compared to $1.5 \mathrm{~T}$ MRI. In addition, in the majority of cases the additional findings of 7.0 T MRI confirmed the unclear lesion on the initial 1.5 T MRI or enabled visualization of the actual lesion, which was not visible at $1.5 \mathrm{~T}$ MRI. Consequently, we suggest that the predictive value of lesion detection on 7.0 T MRI is promising. However, it is important to note that with an increasing spatial resolution the chance of false-positive findings - incidentalomas - also increases. Incidentalomas occur in 10-20\% of the patients, which potentially results in a higher percentage of false-positive findings with the improved lesion detection rate at higher field strengths $[22,23]$.

In addition to pituitary lesion detection, the visualization of the surrounding pituitary anatomy may be used for neurosurgical planning [14, 24-26]. Pituitary gland MRI at 3.0 T has demonstrated to improve the evaluation of the parasellar anatomy and the relation of the lesion to the adjacent structures [14]. However, 7.0 T MRI is expected to provide an even more detailed overview of the parasellar anatomy [27-29]. Nevertheless, with the current retrospective analysis we did not evaluate the parasellar anatomy. Consequently, it was not possible to evaluate the additional value for the performed surgical procedures.

The current study has several limitations. Firstly, a potentially confounding factor is the selection bias that is introduced by only imaging patients at 7.0 T MRI when the $1.5 \mathrm{~T} \mathrm{MRI}$ was inconclusive. This means that only the difficult Cushing's disease cases were included in the current study. Therefore, it is important to notice that a conclusive Cushing's disease diagnosis may be challenging even for an experienced neuroradiologists. Secondly, different imaging protocols were used for clinical $1.5 \mathrm{~T}$ MRI of the pituitary gland because $1.5 \mathrm{~T}$ pituitary imaging was part of routine clinical practice of different referring hospitals. This implies that different vendors of MRI scanners and different scan parameters were used. Furthermore, the clinically acquired sequences were not equal in all patients. This might have influenced our results in favour of the 7.0 T MRI protocol. Still, we feel that the current study gives a realistic view of what can be expected from standard $1.5 \mathrm{~T}$ pituitary gland MRI compared with high resolution MRI at 7.0 T. Thirdly, the reports of the surgical procedure were incomplete in some patients. From these patients it is known whether they were cured after surgery or not. However, it remains unknown from which side of the pituitary gland the microadenoma was removed, hindering a comparison between MRI and surgery in four patients. Fourthly, in two patients no lesion was identified initially on $1.5 \mathrm{~T}$ MRI (Fig. 1) and in two other patients, a lesion was detected initially. However, after consensus reading, a lesion was actually identified in the first two patients, and no lesion was detected in the second two patients. This might indicate that consensus reading could also be a solution for initially undetectable pituitary microadenomas on 1.5 T MRI. Fifthly, a dedicated dynamic contrast enhanced (DCE) sequence was lacking in the current 7.0 T MRI protocol, whereas a DCE sequence at 1.5 T MRI was present in 10 patients. Previous literature demonstrates the clinical advantages of DCE imaging in lesion detection in patients with Cushing's disease [30, 31]. Addition of a DCE sequence with high spatial resolution might increase the detection rate of the smallest lesions. Therefore, a DCE sequence might increase the interobserver agreement as well. Finally, the current retrospective analysis lacks the involvement of 3.0 T MRI. The improvement in SNR and the better spatial resolution by using 3.0 T MRI appears to establish a clear advantage in the localization of microadenomas and in the presurgical planning in patients with Cushing's disease 
$[15,19]$. Still, 3.0 T MRI has not yet supplanted 1.5 T MRI as standard clinical workup for patients with Cushing's disease and a suspected pituitary microadenoma. A large-scale headto-head comparison of 1.5 T, 3.0 T, and 7.0 T MRI with dedicated scanning protocols is needed to evaluate the actual clinical advantages from high spatial resolution MRI in Cushing's disease. A prospective study should include an exact description of the surgical procedure, including the benefit of neurosurgical guidance based on the available detailed MR images.

To conclude, the interobserver agreement of image assessment for 7.0 T MRI in patients with Cushing's disease was good and lesions were detected more accurately with 7.0 T MRI.

Acknowledgments The scientific guarantor of this publication is Jeroen Hendrikse. There is no conflict of interest that could be perceived as prejudicing the impartiality of the research reported. This research did not receive any specific grant from any funding agency in the public, commercial or not-forprofit sector. No complex statistical methods were necessary for this paper. Institutional Review Board approval was obtained. Written informed consent was obtained from all subjects (patients) in this study. Some study subjects or cohorts have been previously reported in European Radiology [16]. Methodology: retrospective, cross sectional study, multicenter study.

Open Access This article is distributed under the terms of the Creative Commons Attribution-NonCommercial 4.0 International License (http:// creativecommons.org/licenses/by-nc/4.0/), which permits any noncommercial use, distribution, and reproduction in any medium, provided you give appropriate credit to the original author(s) and the source, provide a link to the Creative Commons license, and indicate if changes were made.

\section{References}

1. Juszczak A, Grossman A (2013) The management of Cushing's disease - from investigation to treatment. Endokrynol Pol 64:166174

2. Newell-Price J, Trainer P, Besser M, Grossman A (1998) The diagnosis and differential diagnosis of Cushing's syndrome and pseudoCushing's states. Endocr Rev 19:647-672

3. Potts MB, Shah JK, Molinaro AM, Blevins LS, Tyrrell JB, Kunwar $S$ et al (2014) Cavernous and inferior petrosal sinus sampling and dynamic magnetic resonance imaging in the preoperative evaluation of Cushing's disease. J Neurooncol 116:593-600

4. Maio SD, Biswas A, Vezina JL, Hardy J, Mohr G (2012) Pre- and postoperative magnetic resonance imaging appearance of the normal residual pituitary gland following macroadenoma resection: clinical implications. Surg Neurol Int 3:67

5. Witek P, Zielinski G (2012) Predictive value of preoperative magnetic resonance imaging of the pituitary for surgical cure in Cushing's disease. Turk Neurosurg 22:747-752

6. Yamada S, Fukuhara N, Nishioka H, Takeshita A, Inoshita N, Ito J et al (2012) Surgical management and outcomes in patients with Cushing disease with negative pituitary magnetic resonance imaging. World Neurosurg 77:525-532

7. Escourolle H, Abecassis JP, Bertagna X, Guilhaume B, Pariente D, Derome $\mathrm{P}$ et al (1993) Comparison of computerized tomography and magnetic resonance imaging for the examination of the pituitary gland in patients with Cushing's disease. Clin Endocrinol (Oxf) 39:307-313
8. Guy RL, Benn JJ, Ayers AB, Bingham JB, Lowy C, Cox TC et al (1991) A comparison of CT and MRI in the assessment of the pituitary and parasellar region. Clin Radiol 43:156-161

9. Davis WL, Lee JN, King BD, Harnsberger HR (1994) Dynamic contrast-enhanced MR imaging of the pituitary gland with fast spinecho technique. J Magn Reson Imaging 4:509-511

10. Patronas N, Bulakbasi N, Stratakis CA, Lafferty A, Oldfield EH, Doppman J et al (2003) Spoiled gradient recalled acquisition in the steady state technique is superior to conventional postcontrast spin echo technique for magnetic resonance imaging detection of adrenocorticotropin-secreting pituitary tumors. J Clin Endocrinol Metab 88:1565-1569

11. Bartynski WS, Lin L (1997) Dynamic and conventional spin-echo MR of pituitary microlesions. AJNR Am J Neuroradiol 18:965-972

12. Buchfelder M, Nistor R, Fahlbusch R, Huk WJ (1993) The accuracy of CT and MR evaluation of the sella turcica for detection of adrenocorticotropic hormone-secreting adenomas in Cushing disease. AJNR Am J Neuroradiol 14:1183-1190

13. Ludecke DK, Flitsch J, Knappe UJ, Saeger W (2001) Cushing's disease: a surgical view. J Neurooncol 54:151-166

14. Wolfsberger S, Ba-Ssalamah A, Pinker K, Mlynarik V, Czech T, Knosp E et al (2004) Application of three-tesla magnetic resonance imaging for diagnosis and surgery of sellar lesions. J Neurosurg 100:278-286

15. Pinker K, Ba-Ssalamah A, Wolfsberger S, Mlynarik V, Knosp E, Trattnig S (2005) The value of high-field MRI (3T) in the assessment of sellar lesions. Eur J Radiol 54:327-334

16. de Rotte AA, van der Kolk AG, Rutgers D, Zelissen PM, Visser F, Luijten PR et al (2014) Feasibility of high-resolution pituitary MRI at 7.0 tesla. Eur Radiol 24:2005-2011

17. Esposito F, Dusick JR, Cohan P, Moftakhar P, McArthur D, Wang C et al (2006) Clinical review: early morning cortisol levels as a predictor of remission after transsphenoidal surgery for Cushing's disease. J Clin Endocrinol Metab 91:7-13

18. Stobo DB, Lindsay RS, Connell JM, Dunn L, Forbes KP (2011) Initial experience of 3 Tesla versus conventional field strength magnetic resonance imaging of small functioning pituitary tumours. Clin Endocrinol (Oxf) 75:673-677

19. Kim LJ, Lekovic GP, White WL, Karis J (2007) Preliminary Experience with 3-Tesla MRI and Cushing's Disease. Skull Base 17:273-277

20. Bochicchio D, Losa M, Buchfelder M (1995) Factors influencing the immediate and late outcome of Cushing's disease treated by transsphenoidal surgery: a retrospective study by the European Cushing's Disease Survey Group. J Clin Endocrinol Metab 80: 3114-3120

21. Salenave S, Gatta B, Pecheur S, San-Galli F, Visot A, Lasjaunias P et al (2004) Pituitary magnetic resonance imaging findings do not influence surgical outcome in adrenocorticotropin-secreting microadenomas. J Clin Endocrinol Metab 89:3371-3376

22. Hall WA, Luciano MG, Doppman JL, Patronas NJ, Oldfield EH (1994) Pituitary magnetic resonance imaging in normal human volunteers: occult adenomas in the general population. Ann Intern Med $120: 817-820$

23. Scangas GA, Laws ER Jr (2014) Pituitary incidentalomas. Pituitary 17:486-491

24. Mori Y, Kobayashi T, Shibamoto Y (2006) Stereotactic radiosurgery for metastatic tumors in the pituitary gland and the cavernous sinus. J Neurosurg 105 Suppl:37-42

25. Nakazawa H, Shibamoto Y, Tsugawa T, Mori Y, Nishio M, Takami $\mathrm{T}$ et al (2014) Efficacy of magnetic resonance imaging at 3T compared with $1.5 \mathrm{~T}$ in small pituitary tumors for stereotactic radiosurgery planning. Jpn J Radiol 32:22-29

26. Linn J, Peters F, Lummel N, Schankin C, Rachinger W, Brueckmann $\mathrm{H}$ et al (2011) Detailed imaging of the normal anatomy and pathologic conditions of the cavernous region at 3 Tesla 
using a contrast-enhanced MR angiography. Neuroradiology 53: 947-954

27. van der Kolk AG, Hendrikse J, Luijten PR (2012) Ultrahigh-field magnetic resonance imaging: the clinical potential for anatomy, pathogenesis, diagnosis, and treatment planning in brain disease. Neuroimaging Clin N Am 22:343-362

28. Umutlu L, Ladd ME, Forsting M, Lauenstein T (2014) 7 Tesla MR imaging: opportunities and challenges. Röfo 186:121-129

29. Moser E, Stahlberg F, Ladd ME, Trattnig S (2012) 7-T MR-from research to clinical applications? NMR Biomed 25:695-716
30. Tabarin A, Laurent F, Catargi B, Olivier-Puel F, Lescene R, Berge J et al (1998) Comparative evaluation of conventional and dynamic magnetic resonance imaging of the pituitary gland for the diagnosis of Cushing's disease. Clin Endocrinol (Oxf) 49:293-300

31. Kucharczyk W, Bishop JE, Plewes DB, Keller MA, George S (1994) Detection of pituitary microadenomas: comparison of dynamic keyhole fast spin-echo, unenhanced, and conventional contrast-enhanced MR imaging. AJR Am J Roentgenol 163:671679 